\title{
Parachute Aerodynamics from Video Data
}

\author{
Mark Schoenenberger* \\ Eric M. Queen ${ }^{\dagger}$ \\ Juan R. Cruz ${ }^{\ddagger}$ \\ NASA Langley Research Center \\ Hampton, VA 23681
}

\begin{abstract}
A new data analysis technique for the identification of static and dynamic aerodynamic stability coefficients from wind tunnel test video data is presented. This new technique was applied to video data obtained during a parachute wind tunnel test program conducted in support of the Mars Exploration Rover Mission. Total angle-of-attack data obtained from video images were used to determine the static pitching moment curve of the parachute. During the original wind tunnel test program the static pitching moment curve had been determined by forcing the parachute to a specific total angle-of-attack and measuring the forces generated. It is shown with the new technique that this parachute, when free to rotate, trims at an angle-of-attack two degrees lower than was measured during the forcedangle tests. An attempt was also made to extract pitch damping information from the video data. Results suggest that the parachute is dynamically unstable at the static trim point and tends to become dynamically stable away from the trim point. These trends are in agreement with limit-cycle-like behavior observed in the video. However, the chaotic motion of the parachute produced results with large uncertainty bands.
\end{abstract}

\section{$\underline{\text { Nomenclature }}$}

$\begin{array}{llll}C_{m} & \text { Pitching moment coefficient } & m_{a} & \text { Apparent mass, } k g \\ C_{m_{\alpha}} & \text { Pitch moment slope, } \frac{\partial C_{m}}{\partial \dot{\alpha}_{T}} & \mathrm{n} & \text { Number of data points } \\ C_{m_{\dot{\alpha}}} & \text { Pitch damping coeff., } \frac{\partial C_{m}}{\partial\left(\frac{\dot{\alpha}_{T} D_{o}}{2 V}\right)} & P & \text { Pixels } \\ C_{m_{o}} & \text { Linearized } C_{m} \text { intercept } & \bar{q} & \text { Dynamic pressure, } N / m^{2} \\ D_{o} & \text { Reference diameter, } m & R & \text { Parachute length, } m \\ g & \text { Gravitational acceleration, }\left(m / s^{2}\right) & R e & \text { Reynolds number } \\ h & \text { Vertical height, } m & S_{o} & \text { Reference area, } m^{2} \\ I_{y y} & \text { Moment-of-inertia, } k g-m^{2} & t & \text { Time, sec } \\ K & \text { Pixel-per-meter constant } & V & \text { Velocity, } m / s \\ l & \text { Streamwise length, } m & x, y, z & \text { Cartesian coordinates } \\ M & \text { Mach number } & \alpha, \beta & \text { Angle-of-attack, sideslip } \\ \bar{M} & \text { Static moment, } N-m & \alpha_{T} & \text { Total angle-of-attack } \\ m & \text { Parachute mass, } k g & \theta, \psi & \text { Pitch, yaw angles }\end{array}$

${ }^{*}$ Aerospace Engineer, Exploration Systems Engineering Branch, Member AIAA

$\dagger$ Aerospace Engineer, Exploration Systems Engineering Branch, Member AIAA

$¥$ Aerospace Engineer, Exploration Systems Engineering Branch 


$\begin{array}{llll}\rho & \text { Density, } \mathrm{kg} / \mathrm{m}^{3} & y, z & \begin{array}{l}\text { Horizontal, vertical pixels } \\ \text { Freestream }\end{array} \\ \phi & \text { Clock angle } & \infty & \\ \text { Subscripts } & & \text { Superscripts } & \\ 1,2,3 & \text { Calibration point indices } & & \text { Calibration heights, } m \\ a & \text { Apex } & & \\ b & \text { Backshell } & \text { Acronyms } & \\ c & \text { Camera } & \text { DGB } & \text { Disk-gap-band } \\ c p & \text { Center-of-pressure } & \text { MER } & \text { Mars Exploration Rovers } \\ s & \text { Swivel } & \text { MPF } & \text { Mars Pathfinder } \\ p & \text { Pivot } & \text { TDT } & \text { Transonic Dynamics Tunnel }\end{array}$

\section{Introduction}

For the Mars Exploration Rover (MER) mission and other planetary missions using axisymmetric parachutes, the stability and flight characteristics of the parachutes can have a critical impact on terminal descent events prior to landing. The computer algorithms taking data from accelerometer and gyroscopes to control entry descent and landing events (e.g., retro rocket firing) can be adversely affected by erratic motions induced by parachute motions. It is therefore important to understand parachute aerodynamics sufficiently to model this erratic behavior in simulations prior to flight in order to evaluate the robustness of the integrated landing systems in the terminal descent (i.e., under parachute) phase of such missions.

Historically, it has been a very difficult problem to accurately model the behavior of axisymmetric parachutes. While the equations of motion for non-rigid parachutes have been developed, including their dynamic stability, ${ }^{1,2}$ extracting the aerodynamic coefficients experimentally has proven difficult. The aerodynamics from rigid models have provided general characteristics of parachute behavior and sensitivities to geometry. ${ }^{3}$ However, these tests could not address the effects of fabric permeability or non-rigidity of the parachute on the aerodynamics.

Recently, Cruz et al. ${ }^{4}$ tested scale versions of the Viking and Mars Pathfinder disk-gap-band (DGB) parachutes and variants of those parachutes in the NASA Langley Transonic Dynamics Tunnel (TDT). Steady state drag measurements were made with the parachute attached to a swivel at the back of a model MER capsule backshell mounted on a strut in the tunnel in a subsequent test. This provided drag information, but could not explicitly measure stability characteristics. The parachutes were then held fixed in a different test rig and rotated through a sweep of angles-of-attack to extract static pitching moment, normal force and axial force data. While a flight-like fabric parachute was tested, the effects of artificially holding the parachute at a specific angle-of-attack on the static stability measurements could not be quantified. The work presented here attempts to quantify those effects by extracting the pitching moment of one of the parachutes reported by $\mathrm{Cruz}^{4}$ while in the drag test (i.e., free-to-rotate) configuration.

Video was obtained during the free-to-rotate and static tests. Cameras were placed immediately downstream of the parachute looking upstream, and to the side looking across the tunnel. Knowledge of the dimensions of the parachute, tunnel and sting apparatus, as well as the downstream camera position, permits the calculation of angle-of-attack, $\alpha$, and angle-of-sideslip, $\beta$, from the downstream video data of the free-to-rotate tests. This $\alpha, \beta$ information can then be used to extract aerodynamic characteristics of the parachutes tested by the technique described herein.

To better understand and model the motion of DGB parachutes, the video of the free-to-rotate test was used in an attempt to extract quasi-static aerodynamics for comparison with the static data obtained by Cruz. The extraction of dynamic stability derivatives was also performed. This data reduction was performed as a proof-of-concept exercise to develop the technique. The test setup and instrumentation was not selected or designed specifically for this type of parameter identification. It was hoped that static aerodynamics might be extracted with some confidence and evidence of pitch damping functionality with total angle-of-attack might be identified. 


\section{Test Setup}

\section{Tunnel Conditions}

The test setup used by $\mathrm{Cruz}^{4}$ and for the present analysis is shown in Figure 1. Tests were conducted in the NASA TDT. All tests were conducted in air at ambient pressure. For the data set presented here, the freestream Mach number was 0.102 and the dynamic pressure was $736 \mathrm{~N} / \mathrm{m}^{2}$ (15.4 psf). Shown in Figure 1 are the two test configurations. The first setup (Figure 1a) allowed the parachute to move freely in the tunnel and was intended to yield drag data only. Video of the parachute in this configuration is the source of the data set used in the present analysis. The second setup (Figure 1b) held the parachute fixed in a test rig. The rig, extending from a turnable in the tunnel wall holds a parachute at its swivel point as well as its apex. This allows the parachute to be held at a fixed angle-of-attack (adjustable via the turntable) to measure pitching moment, normal force and axial force across a range of angles-of-attack. A ring at the apex permits the canopy to slide in the axial direction as the parachute geometry changes with tunnel conditions and attitude. Video data of one of these tests was used for a calibration of the data extracted from the drag test video.

The parachute used in this work is a scale version of the Mars Pathfinder (MPF) parachute, although material differs from the MPF flight parachute. The test parachute used in this data analysis is F111 Nylon, which is less permeable than the fabric used on the MPF flight parachute. The dimensions and flow conditions of the test are listed in Table 1.

The coordinate system used in the test setup is as follows. The $+x$ axis is aligned with the streamwise flow. The $+y$ axis is horizontal across the tunnel directed towards the left when looking upstream. The $+z$ axis is vertical in the tunnel, directed down (see Figure 1a).

Table 1. MPF-F111 Parachute parameters and tunnel conditions for all data

\begin{tabular}{|c|c|c|c|}
\hline \multicolumn{2}{|r|}{ Parachute Parameters } & \multicolumn{2}{|r|}{ Tunnel Conditions } \\
\hline$D_{o}$ & $1.5816 \mathrm{~m} \quad(5.189 \mathrm{ft})$ & $M$ & 0.106 \\
\hline$S_{o}$ & $1.9554 \mathrm{~m}^{2} \quad\left(21.048 \mathrm{ft}^{2}\right)$ & $\bar{q}$ & $736 \mathrm{~N} / \mathrm{m}^{2} \quad(15.4 \mathrm{psf})$ \\
\hline$R$ & $3.447 m(11.309 \mathrm{ft})$ & $R e$ & $3.7 \times 10^{6 * *}$ \\
\hline$m$ & $0.206 \mathrm{~kg}$ & & \\
\hline$m_{a}$ & $0.294 \mathrm{~kg}$ & & \\
\hline
\end{tabular}

\section{Measurement Apparatus}

The video camera recorded at 29.97 frames per second. Deinterlacing the video signal provided a data frame rate of 59.94 frames per second. As this type of data reduction was not intended at the time of the original testing, the exact position of the camera was not noted. It is known that the camera was located at the same $y$ coordinate in the tunnel as the attachment point of the parachutes. It is also certain that the digital still camera (mounted directly beneath the video camera) was mounted at the same $z$ coordinate as the parachute confluence point. This would place the video camera approximately $0.152 \mathrm{~m}$ (6 inches) above the anchor point. A calibration procedure was developed that independently verified this $0.152 \mathrm{~m}$ offset. The camera's distance downstream of the parachute is fixed at $5.69 \mathrm{~m}$ (224 inches) downstream of the face of the capsule backshell.

\section{Parachute Attitude Extraction}

Figure 2 shows a diagram of the geometry of the parachute in the free-to-rotate test configuration in relation to the downstream camera position. The geometry of this setup was used to calculate the pitch and yaw 


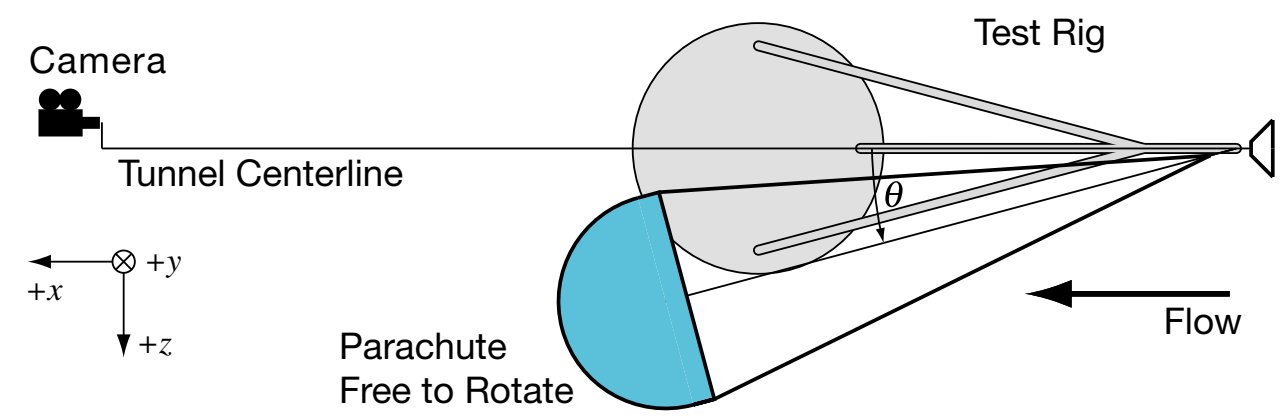

(a) Drag test configuration

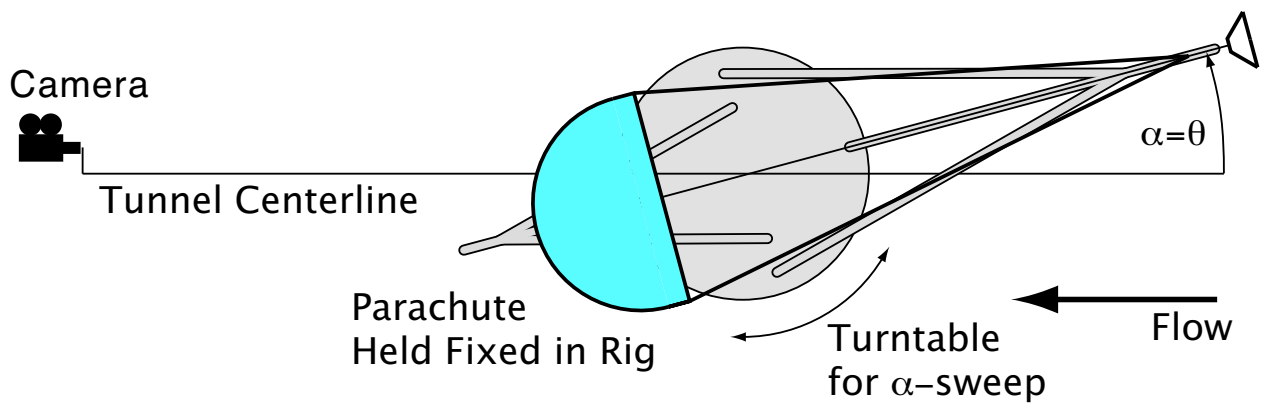

(b) Static aerodynamics test configuration

Figure 1. TDT Parachute testing setup for drag and static aerodynamics testing. Turntable is on left wall of test section as seen looking upstream.

angles, relative to the flow direction, in the $\mathrm{x}-\mathrm{z}$ and $\mathrm{y}-\mathrm{z}$ planes, $\theta$ and $\psi$ respectively. These angles were measured from each frame of the video footage. The instantaneous orientation of the parachute was defined by the position of a line passing through the parachute swivel point and the center of the apex ring. For such a non-rigid body, orientation is difficult to define exactly. However, during the static testing by Cruz, the parachute was held at angle-of-attack at the apex ring, so these two sets of data should be comparable. The apex ring is a metal plug at the apex of the parachute and has a mass of 13.7 grams. This ring was an easily identifiable target in the video footage.

Referring to the geometry in Figure 2, the pitch angle, $\theta$ can be determined from the following equation:

$$
\frac{R \sin \theta+h_{c}}{l_{s}-R \cos \theta}=\frac{h_{a}+h_{c}}{l_{a}}=\frac{h_{b}}{l_{b}}=\frac{P_{z}}{l_{b} K}
$$

Here, $R$ is the length of the parachute from the swivel to the apex, $h_{c}$ is the vertical height of the camera above the swivel, and $l_{s}$ is the axial distance from the swivel to the camera. The quantity, $l_{b}$, is the axial distance from the backshell face to the camera. The constant, $K$, scales the pixels per meter at the axial location of the face of the backshell, and $P_{z}$ is the number of pixels in the video image from the image center to the parachute apex in the vertical direction. Equation 1 does not permit an explicit solution for $\theta$, so an iterative solution was calculated for each data point. The same relation was calculated for $\psi$ except $h_{c}=0$ for the calculations in the $x-y$ plane. Figure 3 shows a captured video image and how the pixels are measured for input into equation 1. For each video frame, the distances from the origin, $P_{y}$ and $P_{z}$, were measured in pixels and converted to the pitch and yaw angles, $\theta$ and $\psi$. 


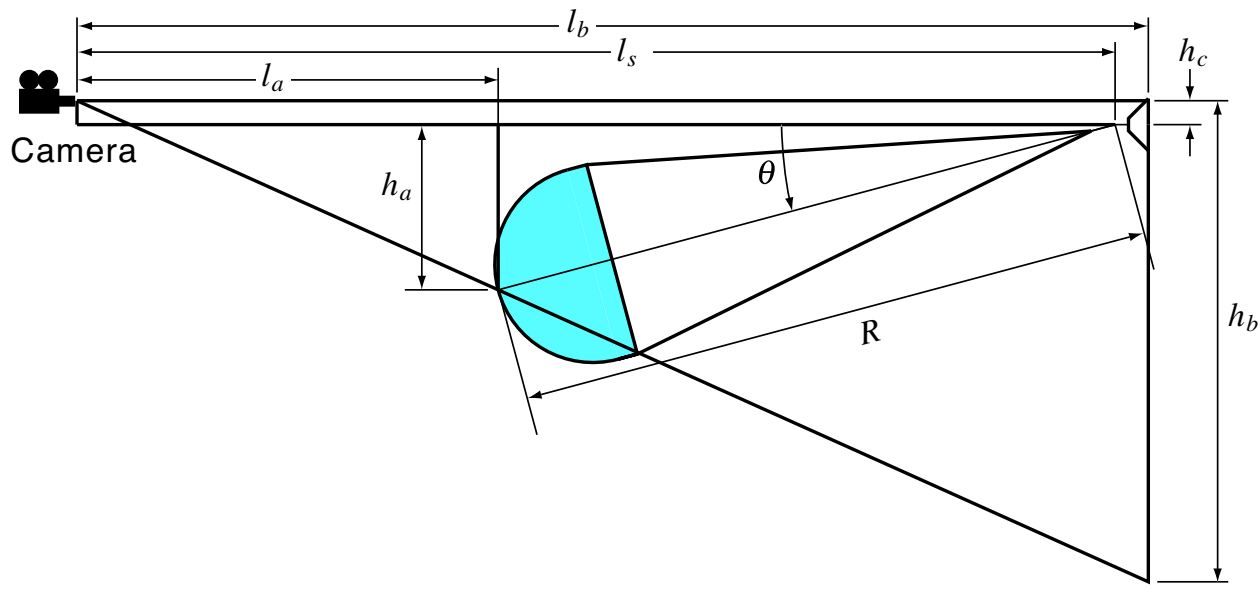

Figure 2. Geometry of video-parachute setup for orientation extraction from free-to-rotate test

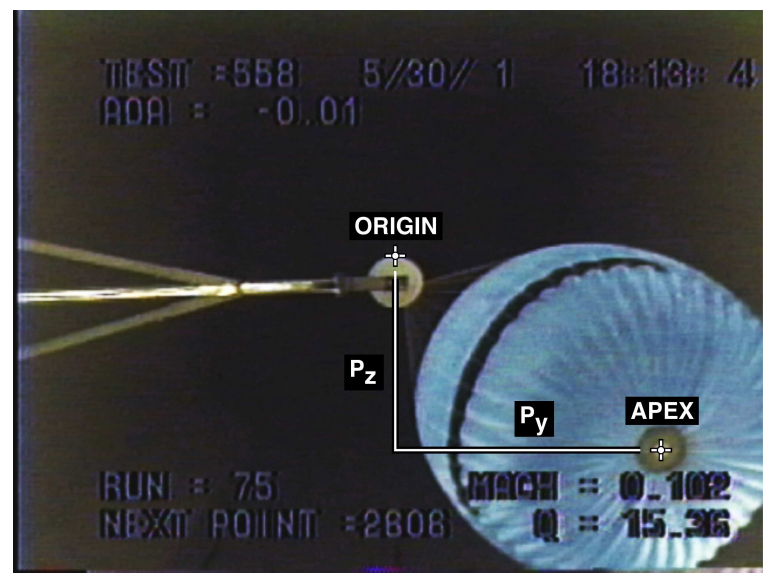

Figure 3. Video image capture example

The angles-of-attack and angles-of-sideslip, $\alpha$ and $\beta$, are then determined from the instantaneous pitch and yaw angles plus components due to the motion of the parachute as it pivots about its anchor point.

$$
\begin{aligned}
& \alpha=\theta+\frac{R_{c p} \cos \theta}{V_{\infty}} \dot{\theta} \\
& \beta=\psi+\frac{R_{c p} \cos \psi}{V_{\infty}} \dot{\psi}
\end{aligned}
$$

Here, $R_{c p}$ is the distance from the swivel point to the parachute center-of-pressure (assumed to be the location of the parachute center of mass) and $V_{\infty}$ is the freestream velocity. For a large freestream velocity, the $\dot{\theta}$ and $\dot{\psi}$ terms are small and the total angle-of-attack can be directly measured from the video data. However, analysis of this test data indicated that $\dot{\theta}$ and $\dot{\psi}$ must be included to obtain the total angle-of-attack that the parachute canopy experiences when it is free to rotate in the tunnel.

For the data reduction described later, these angles were combined into a total total angle-of-attack, $\alpha_{T}$. The total angle-of-attack is calculated from the angles, $\alpha$ and $\beta$, as follows: 


$$
\alpha_{T}=\cos ^{-1}(\cos \alpha \cos \beta)
$$

The azimuthal or clock angle, $\phi$, was also calculated from the pitch and yaw angles to later account for the influence of gravity in the equations of motion. The set of total angle-of-attack data and azimuthal angle formed the raw data from which the stability coefficients were extracted.

\section{Calibration}

To determine the pixels-per-meter constant, $K$, a calibration was performed using video of one of Cruz' static aerodynamic $\alpha$-sweeps. In Cruz' test, the full strut mount, holding a parachute, was swept through a range of angles-of-attack $(-18$ to $+18 \mathrm{deg})$. This provided a good set of images to calibrate the pixels-per-meter constant as there were several reference points that moved through a known path that was very similar to the motions through which the parachute traveled in the free-to-rotate test. Note that for the static test the angle-of-attack, $\alpha$, and the pitch angle in the x-z plane, $\theta$, are identical.

The geometry of the static aerodynamic test setup is shown in Figure 4. Three points were selected for calibration of their observed positions in the video frames to their known positions in the tunnel. These are noted in Figure 4. From the geometry of the test setup, the height of the camera in the tunnel, $h_{c}$, and the pixel-per-meter constant, $K$, were determined from the following three equations which describe the position of the three reference points in the video image.

$$
\begin{aligned}
& \frac{h_{1}^{\prime}}{l_{1}}=\frac{R_{1} \sin \theta+h_{c}}{l_{b}-\left(l_{p}+R_{1} \cos \theta\right)}=\frac{P_{1}}{l_{b} K} \\
& \frac{h_{2}^{\prime}}{l_{2}}=\frac{R_{2} \sin \theta+h_{c}}{l_{b}-\left(l_{p}-R_{2} \cos \theta\right)}=\frac{P_{2}}{l_{b} K} \\
& \frac{h_{3}^{\prime}}{l_{3}}=\frac{R_{3} \sin \theta-h_{c}}{l_{b}-\left(l_{p}+R_{3} \cos \theta\right)}=\frac{P_{3}}{l_{b} K}
\end{aligned}
$$

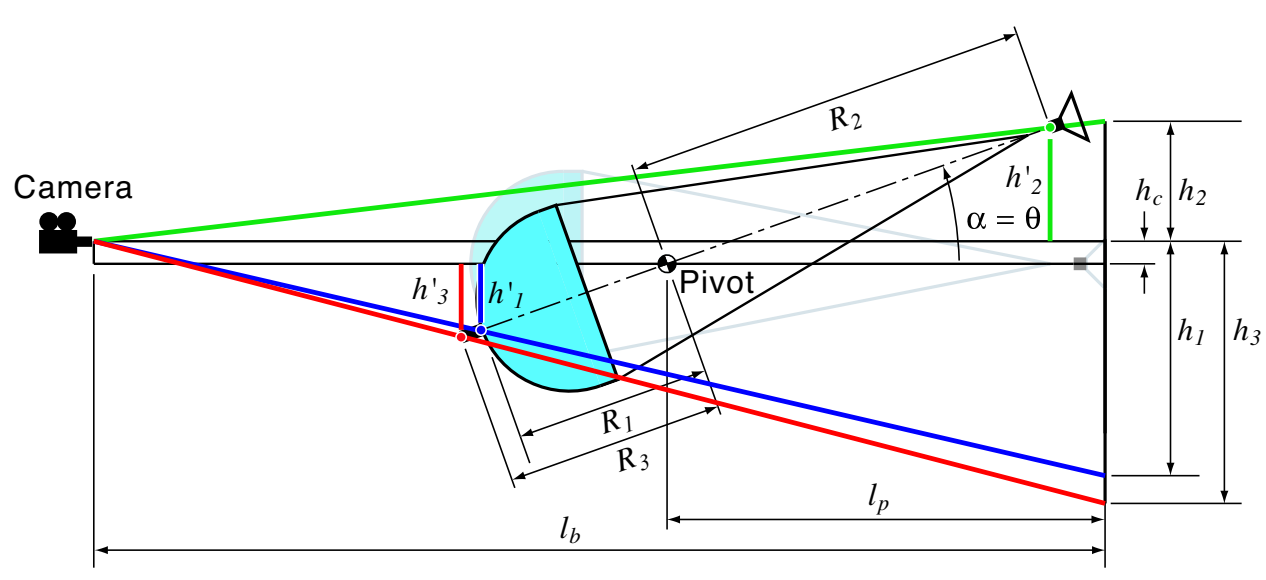

Figure 4. Static test geometry used for calibration

Values for $h_{c}$ and $K$ were determined that best fit the set of these equations at each angle-of-attack measured in the static test. Using three calibration points added confidence that optical distortion of the image was not significant. The values for $h_{c}$ and $K$ were calculated to be $0.1524 \mathrm{~m}(6.0 \mathrm{in})$ and $133.86 \mathrm{pix} / \mathrm{m}(3.40 \mathrm{pix} / \mathrm{in})$ respectively. 


\section{Data Reduction}

\section{Sliding Window Matrix Solution}

To determine the stability coefficients, the time history of the parachute orientation, tracked by the total angle-of-attack, $\alpha_{T}$, and azimuthal angle, $\phi$, was used to extract $C_{m_{\dot{\alpha}}}$ and $C_{m}$ versus total angle-of-attack. For the equations presented here, the derivatives $C_{m_{\dot{\alpha}}}$ and $C_{m_{\alpha}}$ are with respect to total angle-of-attack, $\alpha_{T}$. Other than where needed to account for the influence of gravity, the position in the azimuthal direction was not considered in the data reduction. Due to symmetry, the time-averaged values for the static moment and damping coefficients orthogonal to $\alpha_{T}$ were assumed to be zero.

First, the sum of the moments in the $\alpha_{T}$ plane, about the parachute pivot point, is:

$$
I_{y y} \ddot{\alpha}_{T}-\frac{1}{2} \rho_{\infty} V_{\infty}^{2} S_{o} D_{o}\left[C_{m_{\dot{\alpha}}} \frac{D_{o}}{2 V_{\infty}} \dot{\alpha}_{T}+C_{m_{\alpha}} \alpha_{T}+C_{m_{o}}\right]-m g \cos \phi R_{c p} \cos \alpha_{T}=0
$$

where $g$ is the gravitational acceleration and $R_{c p}$ is the distance from the parachute pivot point to its center of pressure (assumed to be at the same point as the center of mass for this analysis). The clock angle, $\phi$, equals zero when $\psi$ is zero and the $\theta$ is positive. The moment of inertia, $I_{y y}$, about the swivel point is dominated by the combined parachute mass and apparent mass (here assumed to be the air within the fully inflated canopy) acting as a point mass at a distance, $R_{c p}$, from the parachute swivel point. The moment of inertia about the parachute center of mass is small compared to the $\left(m+m_{a}\right) R_{c p}^{2}$ term. Note that the mass, $m$, in the gravity term in Equation 8 is the parachute mass only and does not include the apparent mass.

In Equation 8, the static aerodynamic moment is represented by the linearized expression:

$$
\bar{M}=\frac{1}{2} \rho_{\infty} V_{\infty}^{2} S_{0} D_{0}\left[C_{m_{\alpha}} \alpha_{T}+C_{m_{o}}\right]=\frac{1}{2} \rho_{\infty} V_{\infty}^{2} S_{o} D_{o} C_{m}
$$

where $C_{m_{\alpha}}$ is the local slope of the static moment coefficient curve with respect to total angle-of-attack and $C_{m_{o}}$ is the local intercept of the linearized static moment coefficient curve. Permitting $C_{m_{\alpha}}$ and $C_{m_{o}}$ to vary with the local $\alpha_{T}$ allowed for nonlinear moment curves to be extracted from the video data in this data reduction.

For $\mathrm{n}$ data points from the video frames, $\alpha_{T}$ was measured and second order finite difference values for $\dot{\alpha}_{T}$ and $\ddot{\alpha}_{T}$ (four-point stencils) were calculated to obtain n-4 versions of equation 8 . These were put in matrix form to solve for $C_{m_{\dot{\alpha}}}$ and $C_{m}$ :

$$
\left[\ddot{\alpha}_{T}\right]=\left(\frac{\rho_{\infty} V_{\infty}^{2} S_{o} D_{o}}{2 I_{y y}}\right)\left[\begin{array}{lll}
C_{m_{\dot{\alpha}}} \frac{D_{o}}{2 V_{\infty}} & C_{m_{\alpha}} & C_{m_{o}}
\end{array}\right]\left[\begin{array}{c}
\dot{\alpha}_{T} \\
\alpha_{T} \\
1
\end{array}\right]+\frac{m g R_{c p}}{I_{y y}}\left[\cos \phi \cos \alpha_{T}\right]
$$

Matrix manipulation yields the expression for the moment coefficients:

$\left[\begin{array}{lll}C_{m_{\dot{\alpha}}} \frac{D_{o}}{2 V_{\infty}} & C_{m_{\alpha}} & C_{m_{o}}\end{array}\right]=\left(\frac{2}{\rho_{\infty} V_{\infty}^{2} S_{o} D_{o}}\right)\left(I_{y y}\left[\ddot{\alpha}_{T}\right]-m g R_{c p}\left[\cos \phi \cos \alpha_{T}\right]\right)\left[\begin{array}{c}\dot{\alpha}_{T} \\ \alpha_{T} \\ 1\end{array}\right]^{\top}\left[\left[\begin{array}{c}\dot{\alpha}_{T} \\ \alpha_{T} \\ 1\end{array}\right]\left[\begin{array}{c}\dot{\alpha}_{T} \\ \alpha_{T} \\ 1\end{array}\right]^{\top}\right]^{-1}$

If all data points are used in equation 11, the average values for the stability coefficients across the entire data set are obtained. Provided enough data points are available, it is possible to determine the moment coefficients as a function of $\alpha_{T}$. For this analysis, the data were placed in bins of a specified size according 
to total angle-of-attack. Solving equation 11 for the data in one of these bins finds the moment coefficients at a total angle-of-attack somewhere within the bin limits. For this analysis, the coefficients obtained in a bin are for the mean total angle-of-attack of all the data in that bin.

Continuing with this idea, a sliding bin approach was used to obtain the trends of the moment coefficients across the total angles-of-attack observed in the video data. A bin size was selected (1.5 deg is typical) and placed at the low end of the total angle-of-attack range. For example a bin might contain all points between 7 and $8.5 \mathrm{deg}$. Equation 11 was solved for all the data points in that angle-of-attack range. This yields a set of stability coefficients at the mean angle-of-attack of the points in the bin. The bin was then slid up by an increment, typically smaller than the bin width. For example, the next bin might collect data points between 7.1 and $8.6 \mathrm{deg}$. This new bin contains most of the same points as in the previous bin, losing those at angles below $7.1 \mathrm{deg}$, but adding points between 8.5 and $8.6 \mathrm{deg}$. Equation 11 was solved again to obtain the coefficients at the new mean angle-of-attack and the bin slides over another small increment and so on. Proceeding in this manner, values of $C_{m_{\dot{\alpha}}}$ and $C_{m}$ vs. total angle-of-attack were obtained.

\section{Validation}

To validate this sliding bin parameter identification, a 2-D angle-of-attack history was generated with known moment coefficients. The sliding window data reduction was performed on the angle-of-attack history to extract those known coefficients. The angle-of-attack history was generated with a finite difference simulation of Equation 8. The coefficients in Equation 8 could be selected arbitrarily, but for this case were based on tunnel conditions from the TDT test, the geometry of the MPF F-111 test parachute and the static pitching moment data from the static test program (see Table 1). The static measurements of the F-111 parachute indicated an unstable trim point at zero degrees angle-of-attack and a stable trim point at $\alpha_{T}$ near 12 degrees. The static pitching moment data from Cruz' wind tunnel testing provided a realistic, nonlinear pitching moment coefficient curve to test the parameter identification routine. Figure 5 shows this curve which was used in the validation simulation.

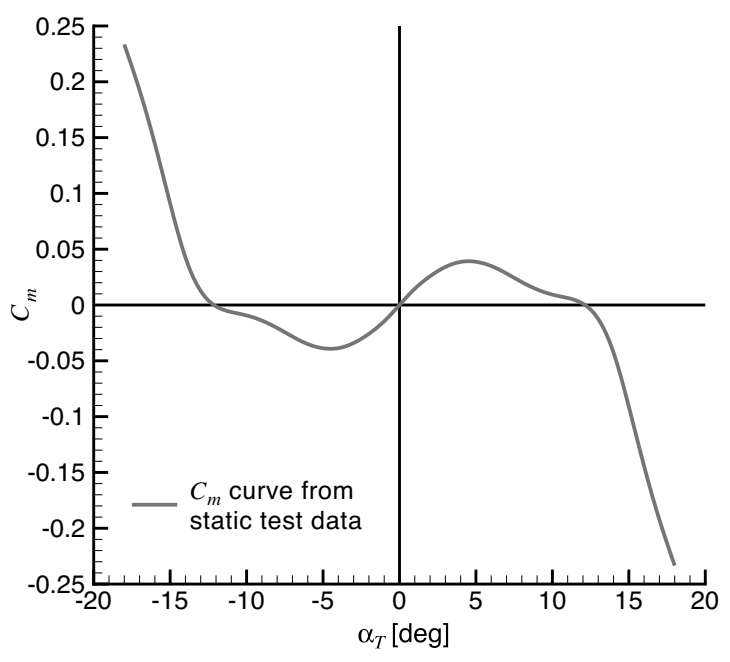

Figure 5. Static pitching moment curve used in validation simulation

To simulate a limit-cycle behavior for the validation simulation only, a simple pitch damping model was created where $C_{m_{\dot{\alpha}}}$ had values of +0.1 from $11<\alpha_{T}<13 \mathrm{deg}$ and -0.1 elsewhere. Figure 6 shows the validation simulation results and indicates that this damping model with the static pitching moment data does produce oscillatory motion that settles into a limit-cycle after an initial displacement from the trim angle-of-attack.

The parameter identification code was then tested by extracting the moment coefficients back out of from the simulation results shown in Figure 6. Figure 7 a compares the extracted $C_{m}$ with the $C_{m}$ curve used 


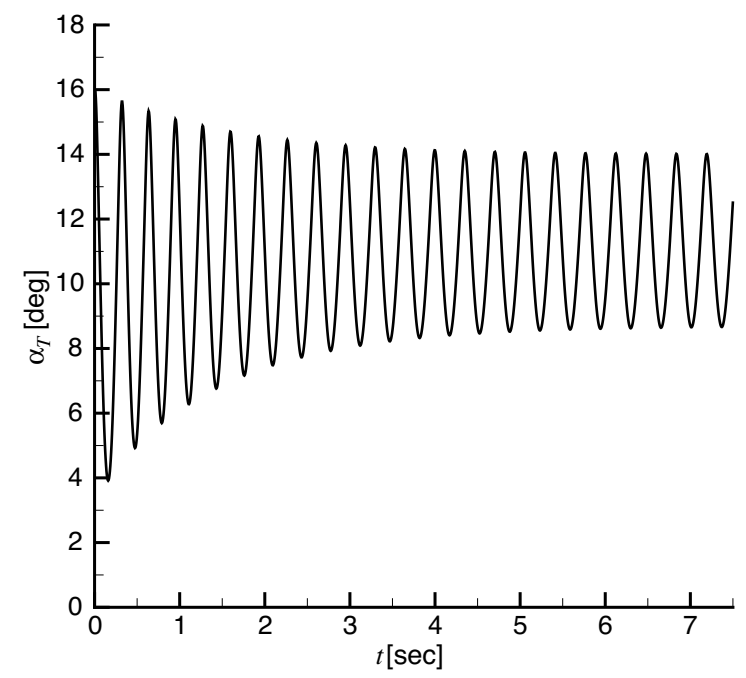

Figure 6. Angle-of-attack time history from validation simulation

in the simulation for several bin sizes. Figure $7 \mathrm{~b}$ shows a similar comparison for $C_{m_{\dot{\alpha}}}$. Figure $7 \mathrm{a}$ shows the parameter identification methodology is very robust to bin size for extracting the pitching moment. The binning technique does have trouble identifying the discontinuity in the simulated pitch damping curve although the errors are reduced with smaller bin sizes. A discontinuous pitch damping model is an extreme case for the parameter identification technique, but illustrates its limitations. For the parameter identification of the parachute video data, a $1.5 \mathrm{deg}$ window was chosen as a compromise between the number of data points reduced in each bin and the smoothing introduced by a wide bin.

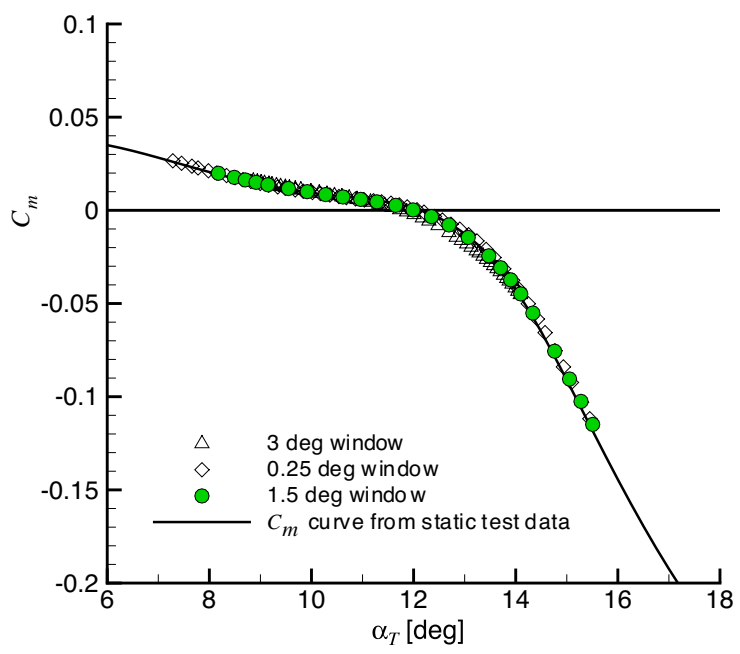

(a) Pitching moment

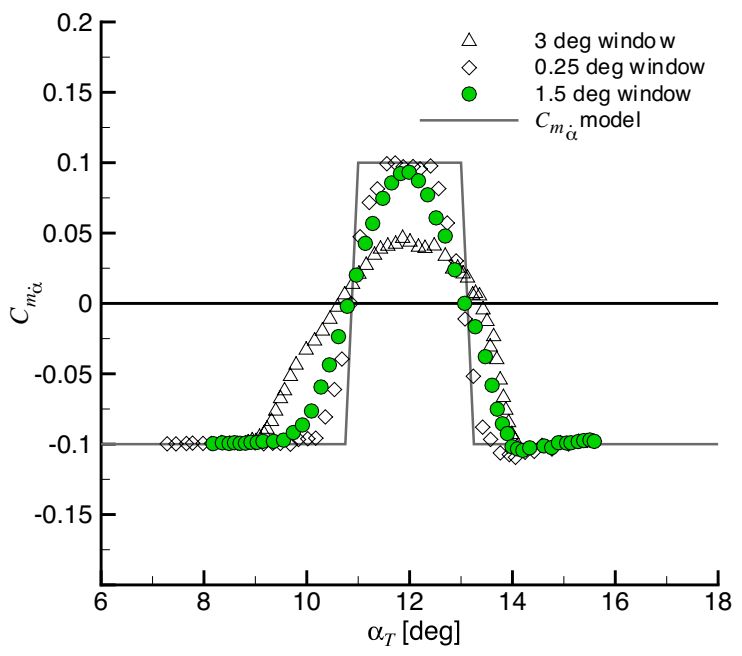

(b) Pitch damping

Figure 7. Aerodynamics extracted from validation simulation against actual simulation values 


\section{Uncertainty Analysis}

To determine estimates of the uncertainties for the dynamic and static moment coefficient results, the data reduction was repeated with perturbed values of the test setup parameters (test apparatus dimensions, pixel-to-meter constant, parachute dimensions etc). The data reduction process was repeated 1000 times with perturbations placed on the key data reduction parameters. Uncertainty values were specified for each parameter and the perturbations were generated randomly with normal distributions within these maximum perturbation values. The results of each of the 1000 perturbed cases were collected and averaged. At each angle-of-attack the mean and standard deviation of the $C_{m}$ and $C_{m_{\dot{\alpha}}}$ values were calculated. The 3-standarddeviation uncertainty bounds are presented in the results section. The maximum perturbation values are listed in Table 2.

Table 2. Perturbation values for uncertainty analysis

\begin{tabular}{cc|cc}
\hline Parameter & Uncertainty & Parameter & Uncertainty \\
\hline \hline$P_{y}$ & \pm 10 pixels & $K$ & \pm 1.969 pix $/ \mathrm{m}(0.05$ pix $/ \mathrm{in})$ \\
$P_{z}$ & \pm 10 pixels & $m_{a}$ & $\pm 0.1 \mathrm{~kg}$ \\
$l_{b}, l_{a}, h_{c}$ & $\pm 0.0254 \mathrm{~m}(1.0 \mathrm{in})$ & $R$ & $\pm 0.1524 \mathrm{~m}(6.0 \mathrm{in})$ \\
\hline
\end{tabular}

\section{Results}

\section{Parachute Attitude Measurements}

Approximately 79 seconds of video data were analyzed, yielding 4761 data points from which pitching moment and pitch damping information was extracted. Figure 8 shows a segment (for clarity) of the parachute's orientation history captured from the video data. The data points are the raw positions extracted from the video. The curve is a local average of the nearest three points along the time history. Comparing the raw data with observations of the video footage, it appeared that the raw data captures some of the motion of the parachute disk relative to the band. This may be exacerbated by the presence of the apex ring. Recall that the apex ring is a significant amount of mass at the apex of the parachute $(6.6 \%$ of the entire parachute/riser mass). The video footage suggests that the relative motion between the disk and band adds high frequency fluctuations to the bulk motion of the parachute. As the apex ring was used in the video analysis to identify the parachute position, this relative motion was captured in the raw data. In an attempt to better represent the parachute motion due to aerodynamic forces, three-point averaged data was used for the parameter identification. Note that the strut extending from the wall to support the backshell model and swivel mount created a wake, disrupting the flow downstream toward the parachute. The parachute tended not to travel through the wake region of the strut. This is apparent in Figure 8 where $\theta \approx 0$ deg and $\psi<0$ deg. Any disruption to the aerodynamic behavior of the parachute caused by the strut's wake was not quantified in the data reduction.

\section{Parameter Identification}

Pitching Moment ( $\alpha_{T}$ plane)

The $1.5 \mathrm{deg}$ sliding window data reduction results for pitching moment are shown in Figure 9. These data show the parachute to be stable and trims at a total angle-of-attack of $10 \mathrm{deg}$. The uncertainty bounds determined from 1000 perturbed cases, shows an uncertainty on the trim angle of approximately $\pm 1.25 \mathrm{deg}$. The parameters that dominate the uncertainties are the parachute length, pixel-per-meter constant and the apparent mass of air in the parachute. Perturbations of the parachute length and the pixel-per-meter constant tend to shift the trim point, while perturbing the apparent mass (which affects the moment of inertia) changes the slope of the pitching moment curve only. 


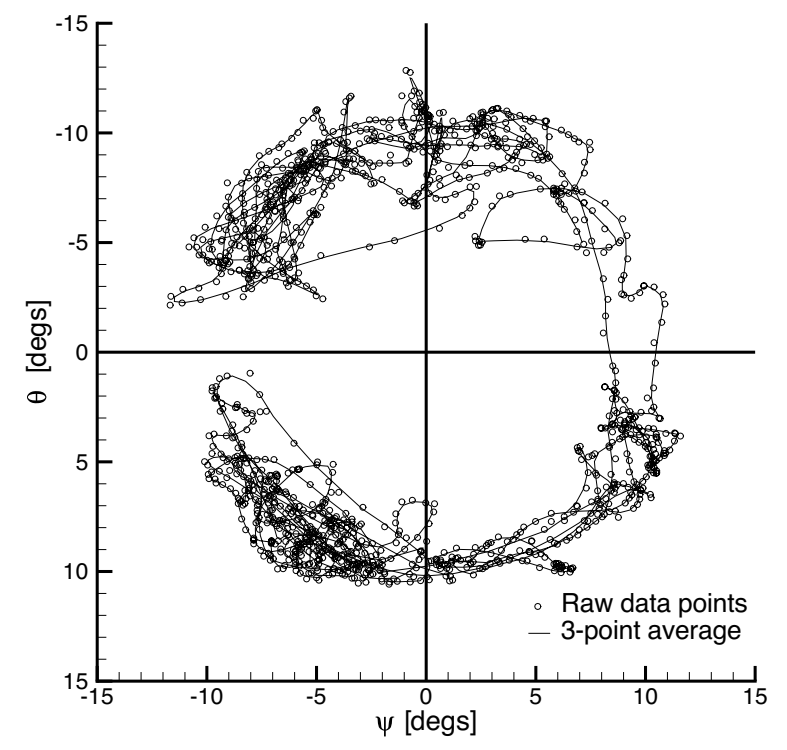

Figure 8. Segment of orientation track

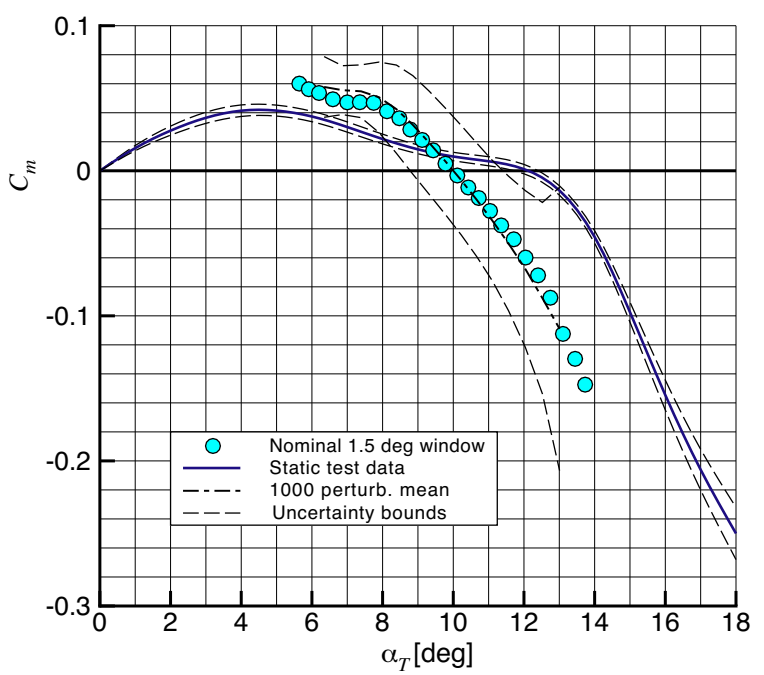

Figure 9. Pitching moment coefficient from $1.5 \mathrm{deg}$ sliding window parameter identification compared to static tunnel data by Cruz. ${ }^{4}$ Moment reference point is at the parachute anchor point.

Figure 9 also shows the static test results (with 3-standard-deviation confidence limits) from Cruz. ${ }^{4}$ Note that the data determined from the video images show the parachute trims 2 degrees shallower than does the static test data. This appears to be a real difference between the parachute's apex-unconstrained behavior and behavior while forced to an angle-of-attack. The difference in trim point is greater than what can be accounted for by uncertainties in each test. This disagreement is not unexpected as significant deformation of the parachute canopy was observed at high angles-of-attack during the static testing. The pitching moment slopes of both sets of data agree fairly well at large angles-of-attack. At total angles-of-attack below the trim point the video-derived pitch damping curve becomes more shallow again agreeing more closely with the slope of the static test data. 
Pitch Damping $\left(\alpha_{T}\right.$ frame)

Figure 10 shows the pitch damping results for 1.5 and 3.0 deg windows. For such chaotic motion of the parachute, it had been hoped that a trend be found for pitch damping as a function of total angle-of-attack. The deformation of the parachute canopy and shedding vortices produce forces and moments that cause dramatic changes in the direction and speed of the parachute, seemingly at random. Even with 79 seconds of data, the $1.5 \mathrm{deg}$ window data reduction does not produce a smooth $C_{m_{\dot{\alpha}}}$ curve. To add data points to each bin (at the expense of angle-of-attack resolution) a $3.0 \mathrm{deg}$ window was used. The $3.0 \mathrm{deg}$ window results do fit within the smaller window data and vary more continuously. Note that a window which encompasses the entire data set would yield one value for $C_{m_{\dot{\alpha}}}$ across this entire total angle-of-attack range. Assuming the parachute has reached a quasi-steady state (e.g., a limit cycle), this would require $C_{m_{\dot{\alpha}}}$ to be zero for the full data set.

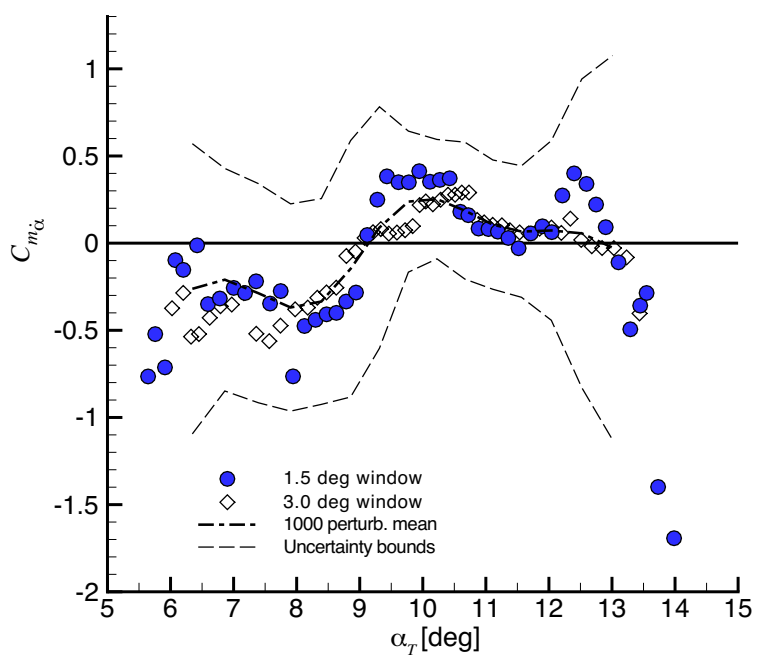

Figure 10. Pitch damping coefficient from parameter identification using 1.5 and 3.0 deg sliding windows. Moment reference point is at the parachute anchor point.

The uncertainty analysis helps understand the apparent trend in pitch damping. Along with the uncertainty bounds, the mean $C_{m_{\dot{\alpha}}}$ values of the 1000 perturbed cases are plotted in Figure 10. This mean curve bisects the 1.5 and $3.0 \mathrm{deg}$ window data and shows a trend with angle-of-attack. At the trim angle-of-attack, the parachute is dynamically unstable $\left(C_{m_{\dot{\alpha}}}>0\right)$. At low angles, the parachute becomes dynamically stable. Above $11.5 \mathrm{deg}$ and below $7 \mathrm{deg}$ total angle-of-attack, the amount of data points drops off significantly and the uncertainty bounds diverge and become quite large. Sets of approximately 2000 equations of the form shown in Equation 8 were used to solve for the stability coefficients (see Equation 11) near the trim angleof-attack, while sets of approximately 100 equations were used at the high and low angle limits of the data. The variation in available data points at each angle-of-attack accounts for the variation in uncertainties. The dynamic stability variation across the entire total angle-of-attack range are small compared to the uncertainties. Therefore, definitive comments about the pitch damping of the parachute are not possible from these data. However, dynamic instability at the trim angle-of-attack coupled with regions of dynamic stability away from the static trim angle is a plausible mechanism for the bounded total angle-of-attack range observed in the free-to-rotate video. Dynamic instability at the trim angle would tend to drive the parachute from equilibrium, while dynamically stable regions away from the trim angle would tend to damp out large motions and the statically stable parachute would return to the trim point.

The bin-averaged pitch damping results are insufficient to replicate the complete behavior of the parachute. The instantaneous pitch damping values can deviate significantly from the bin-averaged data points shown in Figure 10. A more complete model of the parachute behavior may require random components be added to the pitch and yaw damping and static stability coefficients as a function of time. Also, the uncertainties introduced by the selection of bin size remain a concern, although there is fairly good agreement between 
the 1.5 and 3.0 degree window data. Furthermore, the static and dynamic data extracted here provide for no mechanism that would introduce azimuthal motions. A model capable of predicting azimuthal motions that vary in direction and magnitude, while settling to a quasi steady state is required to fully describe the parachute motion. Such a model can not be extracted by the methods developed here, although it should be possible to expand on the principles used in this effort to do so.

\section{Conclusions}

A method for extracting pitching moment versus total angle-of-attack from video data of a parachute that is free to rotate about its anchor point has been developed. Validation work using a simulated oscillating parachute first showed that this parameter identification technique can extract nonlinear static and dynamic pitching moment information from the motion of a 2-D simulation. Next, these parameter identification techniques extracted a pitching moment curve from 79 seconds of video data. These data show the parachute to be statically stable at a total angle-of-attack, in agreement with static wind tunnel tests. The trim angleof-attack, however, was identified to be two degrees shallower than was measured in the static tunnel tests. A study of bin size showed that the 79 seconds of video data were sufficient to capture "bin-converged" static pitching moment characteristics of the parachute.

Although the erratic motion of the parachute suggests that the pitch damping of this parachute fluctuates significantly from instant to instant, the parameter identification results indicate that, averaged over time, the pitch damping varies with angle-of-attack. These data suggest that the parachute is dynamically unstable in the $\alpha_{T}$ plane at the static trim angle-of-attack and becomes dynamically stable as the parachute's attitude departs from this trim angle. Bin-converged solutions were not possible for the dynamic derivatives although the mean curve from uncertainty perturbation cases fits the different bin size results well. Uncertainty analysis indicates that the details of the observed trends are small compared to the uncertainty bounds.

The coefficients extracted with this data reduction technique offer additional information to complement the earlier static testing and free-to-rotate testing. However, these data are still insufficient to fully model the observed motion of the MPF-F111 disk-gap-band parachute. There is no functionality in the static or dynamic coefficients to produce changes in the direction of motion in the azimuthal direction or non-periodic motion in the $\alpha_{T}$ plane. Variations from the time-averaged values of the static pitch and yaw moments and pitch and yaw damping (due to vortex shedding, canopy shape fluctuations, etc.) may all be significant, but cannot be extracted by the methods presented here. Future work should expand the application of this new technique to attempt to quantify the frequency and time variation of the stability coefficients from the time averaged curves identified here. Extracting pitch and yaw stability coefficients with the complete equations of motion may be possible by improving upon this technique, but would require much more data. The technique as presented took advantage of an azimuthal symmetry assumption to extract the stability coefficients as a function of $\alpha_{T}$ only.

This work was done as a proof-of-concept activity. Future parachute tests, with more forethought, may use these techniques to extract static and dynamic stability data with more accuracy. Well placed and clearly identifiable markers located on the parachute, careful calibration of the camera system and accurate measurement of the test setup geometry should yield more accurate and precise results.

Acknowledgements: We would like to especially thank David Petkofsky for his diligent efforts performing video analysis for this work.

\section{References}

\footnotetext{
${ }^{1}$ White, F. M. and Wolf, D. F., "A Theory of Three-Dimensional Parachute Dynamic Stability," Journal of Aircraft, Vol. 5, No. 1,1968 , pp. 86-92.

${ }^{2}$ Wolf, D. F., "Dynamic Stability of a Nonrigid Parachute and Payload System," Journal of Aircraft, Vol. 8, No. 8, 1971, pp. 603-609.

${ }^{3}$ Cockrell, D. J., Huntley, I. D., and Ayres, R. M., "Aerodynamic and Inertial Forces on Model Parachute Canopies," AIAA 75-1371, 1975.

${ }^{4}$ Cruz, J. R., Mineck, R. E., Keller, D. F., and Bobskill, M., "Wind Tunnel Testing of Various Disk-Gap-Band Parachutes," AIAA 2002-2129, 2002.
} 